\title{
An Intersegmental Neuronal Architecture for Spinal Wave Propagation under Deletions
}

\author{
Toni Pérez, ${ }^{1}$ Jesus A. Tapia, ${ }^{4}$ Claudio R. Mirasso, ${ }^{1}$ Jordi García-0jalvo, ${ }^{2}$ Jorge Quevedo, ${ }^{3}$ Carlos A. Cuellar, ${ }^{4}$ \\ and Elias Manjarrez ${ }^{4}$ \\ ${ }^{1}$ Instituto de Física Interdisciplinar y Sistemas Complejos, Consejo Superior de Investigaciones Científicas-Universidad de las Islas Baleares, Campus \\ Universitat de les Illes Balears, E-07122 Palma de Mallorca, Spain, ${ }^{2}$ Departament de Física i Enginyeria Nuclear, Universitat Politècnica de Catalunya, \\ E-08222 Terrassa, Spain, ${ }^{3}$ Departamento de Fisiología, Biofísica y Neurociencias, Centro de Investigación y de Estudios Avanzados, CP 07360 Distrito \\ Federal, Mexico, and ${ }^{4}$ Instituto de Fisiología, Benemérita Universidad Autónoma de Puebla, CP 72570 Puebla, Mexico
}

Recent studies have established and characterized the propagation of traveling electrical waves along the cat spinal cord during scratching, but the neuronal architecture that allows for the persistence of such waves even during periods of absence of bursts of motoneuron activity (deletions) is still unclear. Here we address this problem both theoretically and experimentally. Specifically, we monitored during long lasting periods of time the global electrical activity of spinal neurons during scratching. We found clear deletions of unaltered cycle in extensor activity without associated deletions of the traveling spinal wave. Furthermore, we also found deletions with a perturbed cycle associated with a concomitant absence of the traveling spinal wave. Numerical simulations of an asymmetric two-layer model of a central-pattern generator distributed longitudinally along the spinal cord qualitatively reproduce the sinusoidal traveling waves, and are able to replicate both classes of deletions. We believe these findings shed light into the longitudinal organization of the central-pattern generator networks in the spinal cord.

\section{Introduction}

Scratching is a motor task in which one hind limb performs rhythmic movements to relieve irritating sensations from the skin on the neck, face and pinna. During scratching episodes the cat spinal cord exhibits sinusoidal-like cord dorsum potentials (sinusoidal-CDPs) (Bayev and Kostyuk, 1981). Recently, Cuellar et al. (2009) demonstrated a rostrocaudal propagation of these sinusoidal electrical waves along the spinal cord during fictive scratching in the cat. It is well known that the neuronal circuitry controlling scratching in the cat is located in the hindlimb enlargement of the spinal cord (Sherrington, 1906; Berkinblit et al., 1978; Deliagina et al., 1981; Barajon et al., 1992; Kiehn and Kjaerulff, 1998; Orlovsky et al., 1999). This central pattern generator (CPG) produces the flexor and extensor movements during scratching; however, little is known about its architecture.

To explain the propagating nature of the flexor and extensor activity along the spinal cord, the half-center model of Brown

\footnotetext{
Received April 10, 2009; revised July 9, 2009; accepted July 13, 2009.

This work was supported by the following grants: Consejo Nacional de Ciencia y Tecnología (CONACyT) under project F1-62610 (E.M.), Vicerrectoria de Investigación y Estudios de Posgrado-Benemérita Universidad Autónoma de Puebla (VIEP-BUAP-00070) (E.M.), and Programa Integral de Fortalecimiento Institucional under projects 2008 2009 (E.M.), Mexico; Spanish Ministerio de Educación y Ciencia and Feder under projects FIS2007-60327 (C.M., T.P.) and FIS2006-11452 (J.G.0.); and the European Community under Global Approach to Brain Activity FP6-NEST-043309 project (C.M.,J.G.O.). T.P. acknowledges support from Govern Balear. J.A.T. and C.A.C. acknowledge support from CONACyT Mexico. We thank Pablo Linares from Puebla for technical assistance. Experiments were designed, conducted, and analyzed at the Instituto de Fisiología, Benemérita Universidad Autónoma de Puebla, Puebla, Mexico.

Correspondence should be addressed to Dr. Elias Manjarrez, Instituto de Fisiología, Benemérita Universidad Autónoma de Puebla, 14 Sur 6301, Colonia San Manuel, CP 72570 Puebla, Mexico. E-mail: eliasmanjarrez@gmail.com or emanjar@siu.buap.mx.

DOI:10.1523/JNEUROSCI.1737-09.2009

Copyright $\odot 2009$ Society for Neuroscience $\quad 0270-6474 / 09 / 2910254-10 \$ 15.00 / 0$
}

(1911) was extended to account for a rostrocaudal distribution of neuronal populations along the spinal cord, grouped into modules (in a one-layer architecture) called unit-burst generators (Grillner, 1981). However, this model is not sufficient to explain some aspects of the rhythmic movements, such as deletions (absences of bursts of motoneurons activity) during locomotion or scratching in cats.

A two-layer CPG architecture has been proposed in which a rhythm generation controls the timing, while a second layer provides a mechanism for the pattern formation (Perret and Cabelguen, 1976; Burke et al., 2001; Lafreniere-Roula and McCrea, 2005). This hypothesis is consistent with many experimental observations on deletions during scratching, locomotion, and paw-shake rhythms in the cat (Duysens, 1977; Grillner and Zangger, 1979; Duysens and Pearson, 1980; Grillner, 1981; Perret and Cabelguen, 1976, 1980; Perret at al., 1988; Kriellaars et al., 1994; Burke et al., 2001; Kiehn, 2006). However, that CPG architecture was based on experimental results from one spinal segment. Furthermore, the models associated with this hypothesis did not consider longitudinal coupling between CPG neuronal circuits along the spinal cord, and thus could not provide a mechanism for the propagation of the scratching activity through deletion periods.

Deletions can be classified as resetting or non-resetting, depending on whether the oscillation phase is altered or not during the deletion (Rybak et al., 2006b). A resetting deletion is characterized by a phase shift of the post-deletion rhythm with respect to the pre-deletion oscillations. In contrast, after a non-resetting deletion the phase of the pre-deletion rhythm is not shifted after the deletion. The first aim of the present research was to show that the sinusoidal-CDPs are maintained during a non-resetting de- 
A

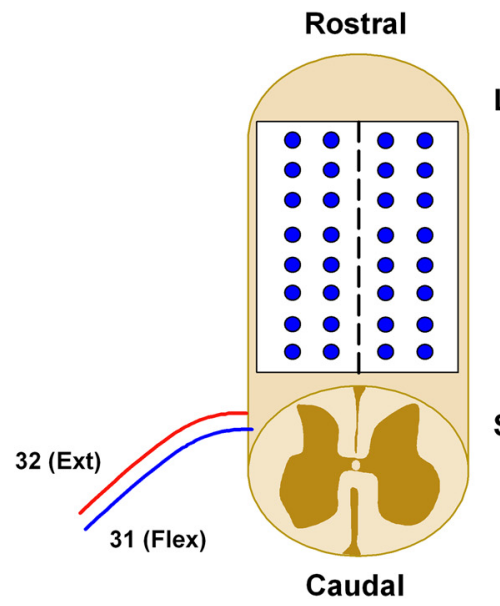

B

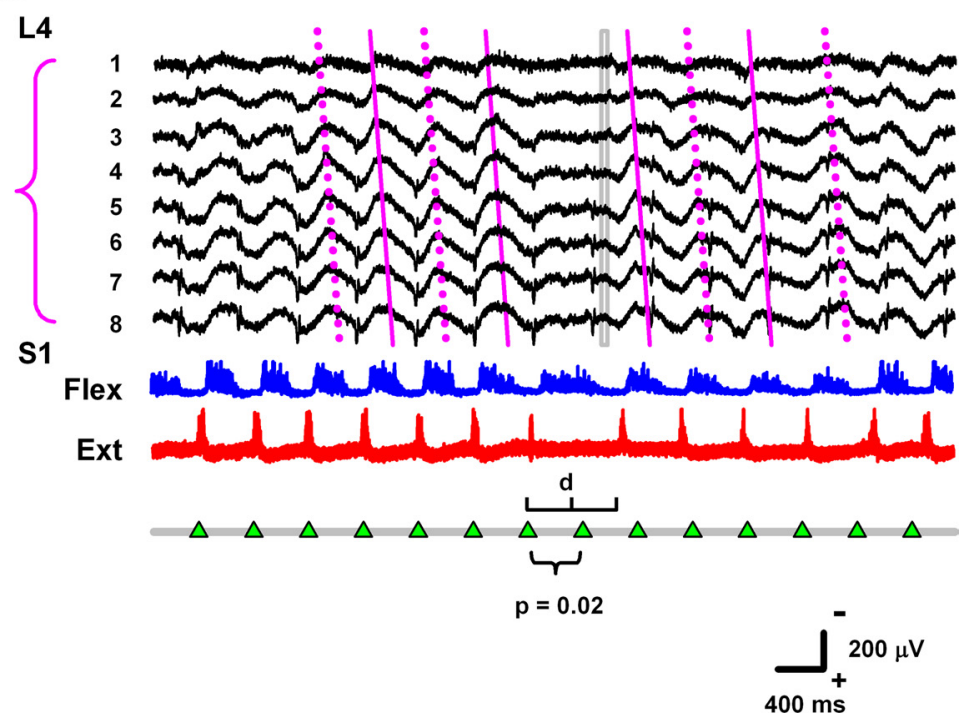

Figure 1. $\quad \boldsymbol{A}$, Scheme of the experimental arrangement. $\boldsymbol{B}$, Recordings of spinal traveling waves and electroneurograms during scratching. The traces illustrate one resetting deletion (d) of the extensor MG motoneuronal activity. The magenta lines illustrate the rostrocaudal propagation of the CDP waves from electrodes 1-8. Flex and Ext indicate the activity of the flexor TA, and extensor, MG nerves, respectively. The arrowheads are spaced at the mean pre-deletion period (average of 5 periods). Intervals on the black horizontal bar were calculated from the hypothesized number of cycles present in the deletion. The interval spanning the deletion dis significantly different from the control (mean pre-deletion period) $(p<0.05)$. Note the alteration in the phase for the resetting deletion d even after the post-deletion period. Furthermore, note that the deletion interval $d$ is not a multiple integer of the mean pre-deletion period. The recordings show that during the resetting deletion the sinusoidal CDP is abolished. The vertical rectangle shows the end of the resetting phase.

letion, but temporally disappear during a resetting deletion of the extensor motoneurons. The second aim was to interpret these data in terms of a theoretical model based on a two layer longitudinal architecture of the CPG, and to propose a theoretical circuit that reproduces the sinusoidal shape of the CDPs. In this context, our study extends, both experimentally and theoretically, the longitudinal analysis of the CPG organization at the level of spinal interneurons.

\section{Materials and Methods}

The analysis of deletions was investigated in the same animals used in a recent paper (Cuellar et al., 2009), as well as in other cats. In that study we described the preparation as well as the techniques of multielectrode recording.

Preparation. Experiments were performed in 18 adult cats $(2.0-3.5$ $\mathrm{kg}$ ). For surgery, anesthesia was induced and maintained with halothane $(1 \%)$ delivered in a mixture of $30 \%$ oxygen and $70 \%$ nitrous oxide. Atropine $\left(0.05 \mathrm{mg} \mathrm{kg}^{-1}\right)$ and dexamethasone $\left(2 \mathrm{mg} \mathrm{kg}^{-1}\right)$ were given at the beginning of surgery. The radial vein was canulated to administer fluids and the carotid artery to monitor blood pressure. A bicarbonate (100 $\mathrm{mm}$ ) and glucose (5\%) solution was delivered intravenously throughout the experiment at a rate of $5 \mathrm{ml} \mathrm{h}^{-1}$. Dextran and saline solutions were given as necessary to maintain blood pressure between 80 and $120 \mathrm{mmHg}$. Guidelines contained in the National Institutes of Health Guide for the Care and Use of Laboratory Animals (85-23, revised in 1985) were strictly followed. The level of anesthesia was verified throughout the surgery by monitoring arterial blood pressure and by testing for the lack of withdrawal reflexes and muscle tone.

Electrophysiological recordings. Fictive scratching was provoked by tactile stimulation of scratch reflex receptive fields located around the pinna, neck or the head, after the application of a piece of cotton impregnated with D-tubocurarine $(0.1 \%)$ on the surface of the cervical C1-C2 spinal segments.

We recorded electroneurograms (ENGs) from the distal end of the following sectioned flexor (Flex) and extensor (Ext) nerves: tibialis anterior (TA: Flex), lateral gastrocnemius plus soleus (LGS: Ext) or medial gastrocnemius (MG: Ext) nerves. Cord dorsum potentials were monopolarly recorded (in DC or AC mode) on a Synamps electroencephalographic (EEG) amplifier (NeuroScan) using a system of $30 \mathrm{Ag}$-AgCl electrodes ( $200 \mu \mathrm{m}$ diameter) positioned on the surface of the lumbo-sacral L4 to S1 spinal cord against an indifferent electrode placed on the paravertebral muscles, as illustrated in Figure $1 \mathrm{~A}$ (for clarity, in the present study we are illustrating recordings only from electrodes $1-8$ ). The distance between electrodes was $5 \mathrm{~mm}$ (rostrocaudal) and $1 \mathrm{~mm}$ (mediolateral). A careful identification of the L4 to S1 ventral roots allowed the precise positioning of the multielectrode array on the L4-S1 spinal segments (Cuellar et al., 2009). Sinusoidal-CDP waves represent the rhythmic activity of dorsal horn and intermediate zone interneurons. This is supported by the fact that sinusoidal waves during scratching are barely affected after lesion of motor nuclei (L4-S2) along the recorded segments (Cuellar et al., 2009).

Statistical analysis to examine changes in cycle period and phase during deletions. We performed a statistical analysis to examine significance of possible changes in cycle period and phase during deletions. Such statistical analysis was performed as in other studies that examined changes in cycle period during deletions (for details, see Lafreniere-Roula and McCrea, 2005). The motivation of this analysis was to check whether cycle duration and phase of the sinusoidal-CDP remains unaltered during deletions in the activity of the LGS or MG nerves.

The cycle period was measured between burst onsets in the ENG recording. Cycle intervals (periods) during deletions were compared with cycle intervals immediately preceding the deletion. The $t$-statistic (Sokal and Rohlf, 1969) was used to evaluate the variation in cycle period between the deletion and the average of 5 consecutive cycles preceding the deletion. The hypothesized number of deletions was based on the number of predicted rhythmic bursts in the ENG of the nerves. Furthermore, a hypothesis was made that the deleted activity spanned an integer number of cycles $(1,2$, or 3$)$. The $t$-statistic was used to calculate the $p$ 
value, with a $p$ value $>0.05$ meaning that the cycle intervals during deletions were not significantly different to cycles before the deletion (the cycle period did not change).

We performed a similar analysis for the phase of the cycle periods. Statistically significant changes $(p<0.05)$ in the phase of cycle periods were used to define the perturbation as a resetting deletion; in contrast, nonstatistically significant changes $(p>0.05)$ in the phase were used to consider the perturbation as a non-resetting deletion. In this context, a resetting deletion is one in which the bursting is either phase-advanced or phase-delayed, whereas a non-resetting deletion means that there is no change in the phase. That is, if one were to plot the onset time of bursts in the ENG, the burst after the non-resetting deletion would follow the burst preceding the deletion by an integer multiple of the pre-deletion interburst interval (i.e., a non-resetting deletion). If this is not an integer multiple, the period postdeletion would be shorter or larger than control periods (a resetting deletion).

\section{Results}

A previous study (Cuellar et al., 2009) analyzed in detail the phenomenon of propagation of electrical waves (sinusoidal CDPs) on the cat spinal cord during scratching. In the present study we experimentally analyze these electrical waves during the spontaneous occurrence of resetting or non-resetting deletions of extensor activity, and give a theoretical support for their occurrence. Figure $1 B$ shows recordings of CDPs and of the extensor and flexor nerve activities during a long-lasting perturbation, which includes a typical resetting deletion of the MG extensor activity ( $d$, bottom trace). Note that during this deletion there is a concomitant absence of sinusoidal CDPs and a change in phase of the nerve activities after the deletion. The change in phase can be visualized from the evenly spaced triangles at the bottom of Figure $1 \mathrm{~B}$. Furthermore, note the propagating nature of the sinusoidal CDPs (magenta lines) before and after the perturbation.

Recent studies of the CPG architecture (Lafreniere-Roula and McCrea, 2005; Rybak et al., 2006a,b; Kiehn, 2006) predicted the existence of a set of CPG neurons that could maintain the cycle timing during non-resetting deletions in the extensor motoneurons. Here we present the first experimental evidence that would confirm that prediction. We observed that the sinusoid-like spinal waves persist during non-resetting deletions of extensor nerves, thus suggesting the existence of a population of neurons belonging to the CPG network that are able to maintain the rhythm during deletions. Figure 2 shows recordings of the CDPs and of the extensor and flexor nerves when a non-resetting deletion in the MG extensor activity occurred. Furthermore, note the propagating nature of the sinusoidal CDPs (superimposed lines) before, during and after the perturbation. Because the period and phase of the extensor activity were unaltered during deletions, this kind of deletion was considered as non-resetting. Remarkably, the traveling waves were also unaltered during this type of deletion.

Figure 3 shows a more detailed analysis of the resetting and non-resetting deletions that occur during a traveling wave. For clarity, in this figure we only selected the recordings from electrodes 3 and 5 . Note the phase gradient in these sinusoid-like
CDP oscillations, thus indicating the existence of a traveling wave. The horizontal rectangle in Figure $3 A$ indicates the absence of sinusoidal traveling waves during an extensor resetting deletion (black vertical arrow). The dots in the four graphs below the recordings show the corresponding cycle periods in one cat. Note also the absence of CDP oscillation during the deletion in the extensor nerve. The abscissas in the histograms shown in the bottom plots of Figure $3 A$ represent the statistical significance $p$ level obtained by the Student's $t$ test applied to determine potential differences in phase and cycle period before and after the deletion (see Materials and Methods). We observed these deletions in the extensor activity in 14 of 20 cats (42 episodes). We obtained $p$ values $<0.05$, thus indicating that the deletions of extensor activity observed during the propagation of the CDP wave belonged to the resetting type in this case.

The arrow in Figure $3 B$ indicates the time in which a nonresetting deletion in the extensor activity occurs. The dots in the four graphs below the recordings show the corresponding cycle periods in one cat. Both the CDP oscillation and the burst of flexor activity persist during the deletion in the extensor nerve. Note the lack of activity of flexor motoneurons during the inactive phase due to normal alternation. We observed these deletions in the extensor activity in 17 of 20 cats ( 73 episodes). The histograms in Figure $3 B$ show the statistical analysis of the differences between the means of the phase and cycle period right before the deletion and during the deletion. We obtained $p$ values $>0.05$ in 73 of 115 deletions, thus indicating that the deletions of extensor activity observed during the propagation of the CDP wave were mainly non-resetting in this case.

For clarity, we also compared systematically the mean cycle period preceding the deletion versus the estimated period during the deletion. Figure $4 \mathrm{~A}$ (left plot) shows that during resetting deletions, the cycle period during the deletion falls outside of the SD of the cycle period preceding the deletion (i.e., the cycle period does change). Such a cycle period during the resetting deletion is not a multiple integer of the pre-deletion period. In contrast, Figure $4 A$ (right plot) shows that during non-resetting deletions the cycle period during the deletion falls within the SD 
A

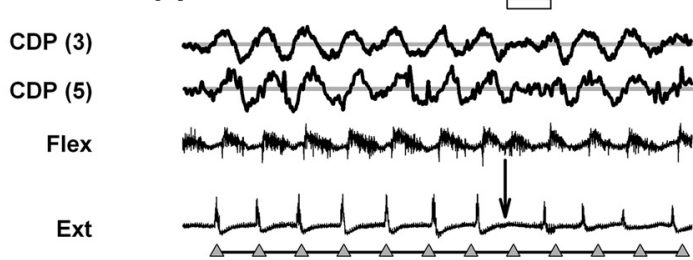

Cycle period (s)

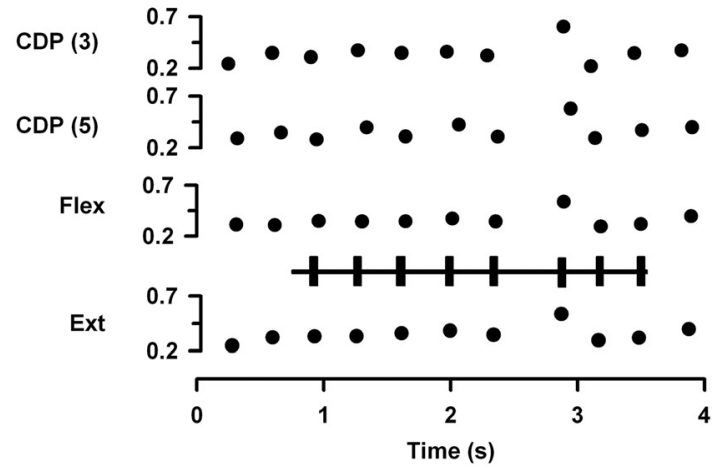

N
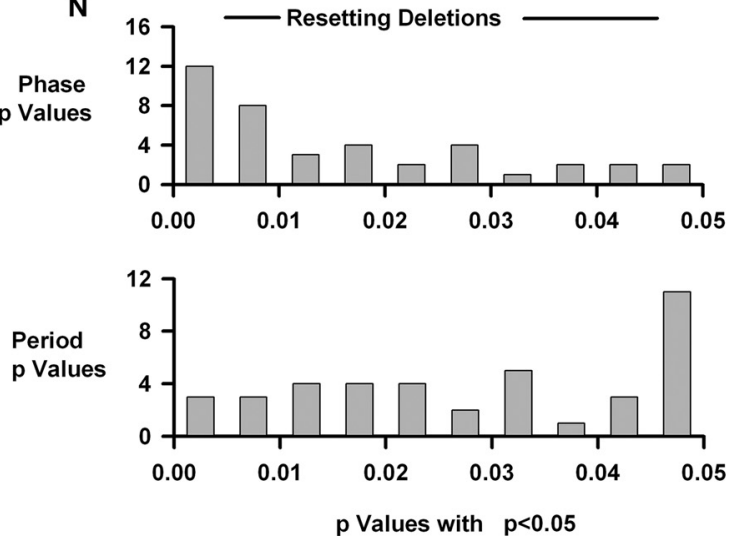

B
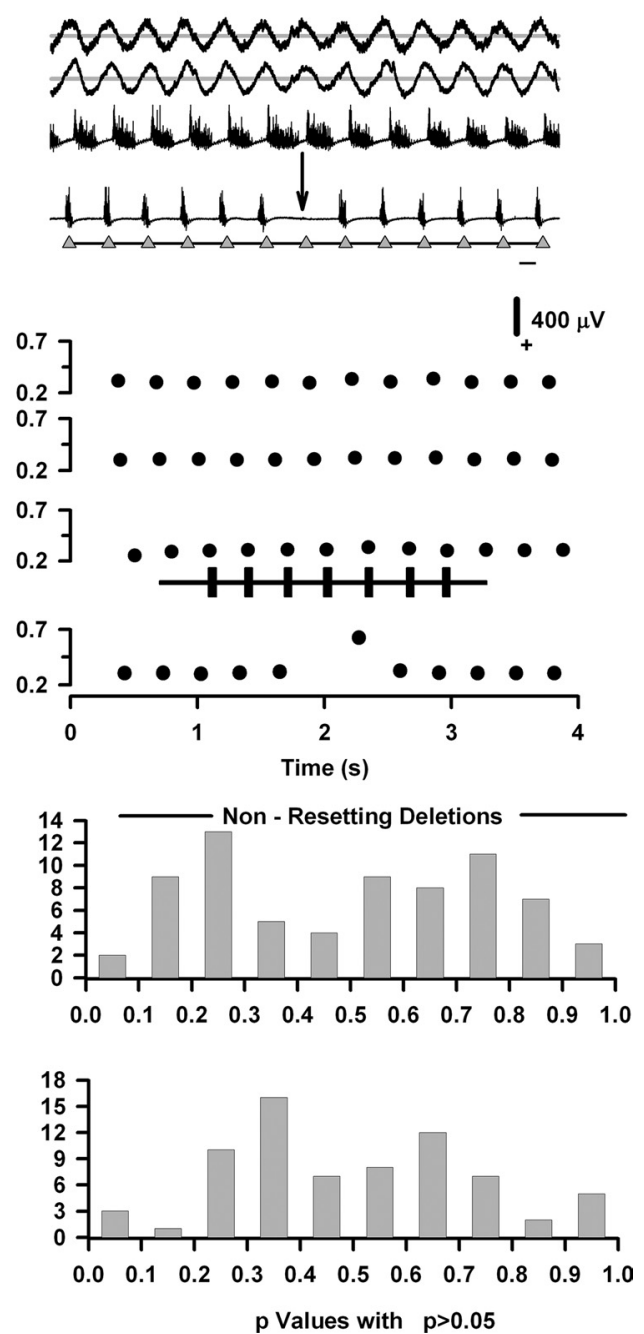

Figure 3. $\boldsymbol{A}$, Absence of sinusoidal CDPs during resetting deletions in the activity of the MG nerve. Bottom, histograms of $p$ values. The $p$ value indicates the statistical significance of the difference between the phase (and cycle periods) before and during the deletion. These values were obtained by the statistical significance of the difference between the phase (and cycle periods) immediately preceding the deletion and the phase (and cycle periods) during deletions. $p<0.05$ indicates resetting deletions. $\boldsymbol{B}$, The same as $\boldsymbol{A}$ but for non-resetting deletions. In this case $p>0.05$ indicates a non-resetting deletion. Data obtained from the analysis of 15 cats. The arrows indicate the occurrence of the deletions.

of the cycle period preceding the deletion (the cycle period does not change).

Figure $4 B$ (left plot) shows that during resetting deletions, the phase during the deletion falls outside of the SD of the phase preceding the deletion (the phase does change). In contrast, Figure $4 B$ (right plot) shows that during non-resetting deletions the phase during the deletion falls within the SD of the phase preceding the deletion (the phase does not change). Interestingly, Figure $4 C$ (left plot) shows that during resetting deletions, the sinusoidal-CDP amplitude (measured from peak to peak) during the deletion is significantly abolished. In contrast, Figure $4 C$ (right plot) shows that during non-resetting deletions the sinusoidal-CDP amplitude during the deletion does not change.

Figure $4 D$ shows that up to 4 consecutive resetting (or nonresetting) deletions can occur during each perturbation. We have recorded episodes in which there is an elimination of the CDP spinal wave when a short (of 1 or $<1$ cycle period) perturbation occurs (Fig. 3A). Oppositely, we have recorded episodes in which there is no elimination of the CDP spinal wave even if the non- resetting perturbation is of long duration. Thus, the elimination of the CDP spinal wave is associated with resetting perturbations regardless of the number of deletions.

In summary, we found similar results both for the phase and the period analysis. We obtained statistically significant changes in the phase of cycle periods for resetting deletions $(p<0.05)$, but not for non-resetting deletions $(p>0.05)$ (Figs. 3, 4).

\section{The model}

To gain insight into the experimental findings, we modeled the rhythm generation and the signal propagation along the spinal cord after the scheme shown in Figure 5A. The network involved in the propagation of scratching activity across the segment L4-S1 of the spinal cord consists of eight CPG units (or "nodes") (Grillner, 2006), chosen to match experimental measurements. These CPGs were connected via excitatory synapses with their nearest neighbors along the rostrocaudal direction. Each CPG unit contains neurons and interneurons connected in a similar manner as discussed by Brownstone and Wilson (2008). We considered the CPG network as consisting of a two-level 
architecture containing a half-center rhythm generator (RG) and a pattern formation (PF) network, as suggested by Rybak et al. (2006b). The RG, formed by two populations of excitatory neurons that mutually project on each other via inhibitory interneuron populations (Fig. 5A, gray and small red balls, respectively), is responsible for the duration of the flexor and extensor phases (defining the scratching rhythm). It is also responsible for controlling the activity of the PF network by exciting the flexor and inhibiting the extensor half-center of the PF network. The flexor and extensor half-centers of the PF network consist of excitatory neuron populations that form reciprocal connections with one another through inhibitory interneuron populations. Based on the experimental evidence that rhythmic bursting activity in flexor motoneurons can continue in the absence of extensor burst (Pearson and Iles, 1970; Pearson, 1972) we connected the RG layer and the PF network through an asymmetric direct excitatory synapse on flexor PF neurons population and through an interposed inhibitory interneuron population on extensor PF neuron population (Brownstone and Wilson, 2008). To keep the model simple (but still realistic) we assumed each population to be composed of 20 neurons described by a modified Morris-Lecar model (Rinzel and Ermentrout, 1998). We included heterogeneity within each neuron populations by a random distribution of the leakage current reversal potential $V_{\mathrm{L}}$. Connections between populations were set such that individual neurons of each population receive $15 \%$ of randomly chosen connections from the other population. Excitatory synaptic connections from populations within the PF layer directly excite the motoneuron populations (see, for example, connections from populations 3 and 4 to extensor and flexor motoneuron populations, respectively, in Fig. $5 A$ ). In addition, reciprocal inhibitory synaptic connections via inhibitory interneuron populations between the excitatory neuron populations, and flexor and extensor motoneuron populations were also considered. Excitatory synaptic connections were also considered between adjacent rostrocaudal elements along the propagation axis (Fig. 5A; light gray arrows represent synapses between the CPGs).

\section{Model and numerical results}

In this section we describe in more detail the model we used to study the rhythm generation and signal propagation along the spinal cord. To model the neuronal activity within the CPG we used a square-bursting version of the Morris-Lecar

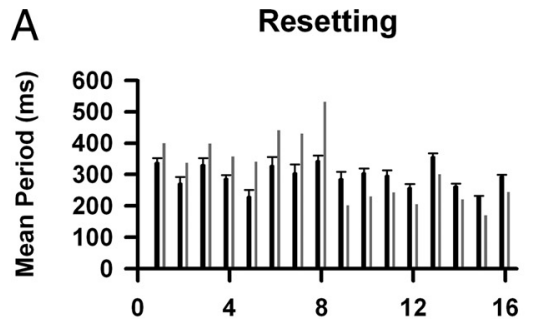

Non-Resetting
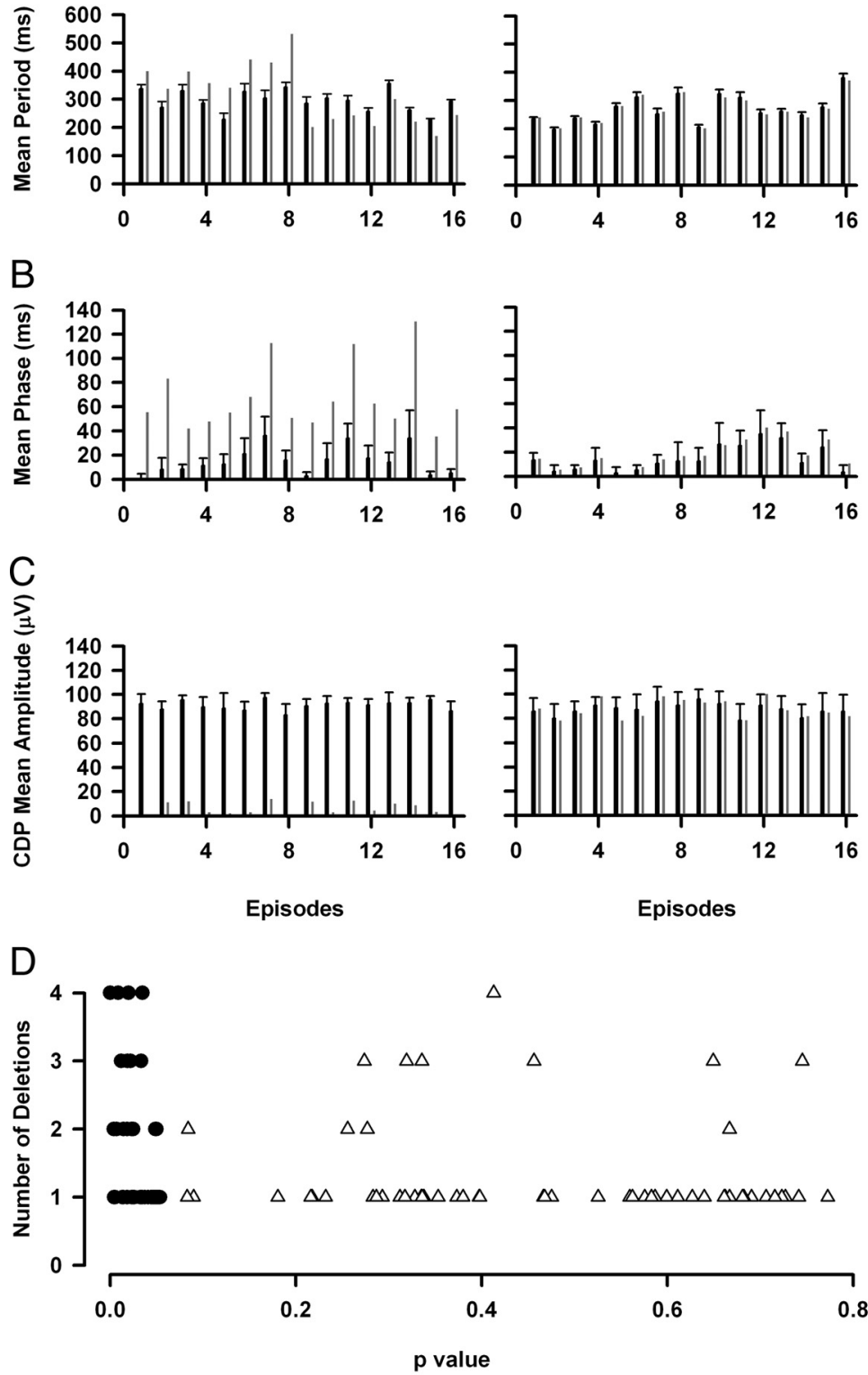

- Resetting Deletions ( $p<0.05)$

$\Delta \quad$ Non-Resetting Deletions $(p>0.05)$

Figure 4. A, Left plot, averaged cycle period preceding the resetting deletions (black bars) versus the cycle period during the resetting deletion (gray bars). $\boldsymbol{B}$, Right plot, The same as the left plot, but for non-resetting deletions. $\boldsymbol{B}$, The same as $\boldsymbol{A}$, but for the mean phase. $C$, The same as $A$, but for the mean amplitude of the sinusoidal CDPs (measured from peak to peak). Error bars indicate the SD for 5 cycles. The numbers in the horizontal axis indicate different scratching episodes. $\boldsymbol{D}$, Number of deletions versus $p$ value to compare the mean cycle period of the extensor activity preceding the deletion and the cycle period of the hypothetical absence of extensor activity during the deletion (see Materials and Methods).

model (Rinzel and Ermentrout, 1998). The equations read as follows:

$$
\begin{gathered}
C \frac{d v}{d t}=I-g_{\mathrm{Ca}} m_{\infty}(v)\left(v-V_{\mathrm{Ca}}\right) \\
-g_{\mathrm{K}} w\left(v-V_{\mathrm{K}}\right)-g_{\mathrm{L}}\left(v-V_{\mathrm{L}}\right) \\
\quad-g_{\mathrm{KCa}} z(y)\left(v-V_{\mathrm{K}}\right)-I^{\mathrm{syn}} \\
\frac{d w}{d t}=\phi \tau(v)\left(w_{\infty}(v)-w\right)
\end{gathered}
$$


A

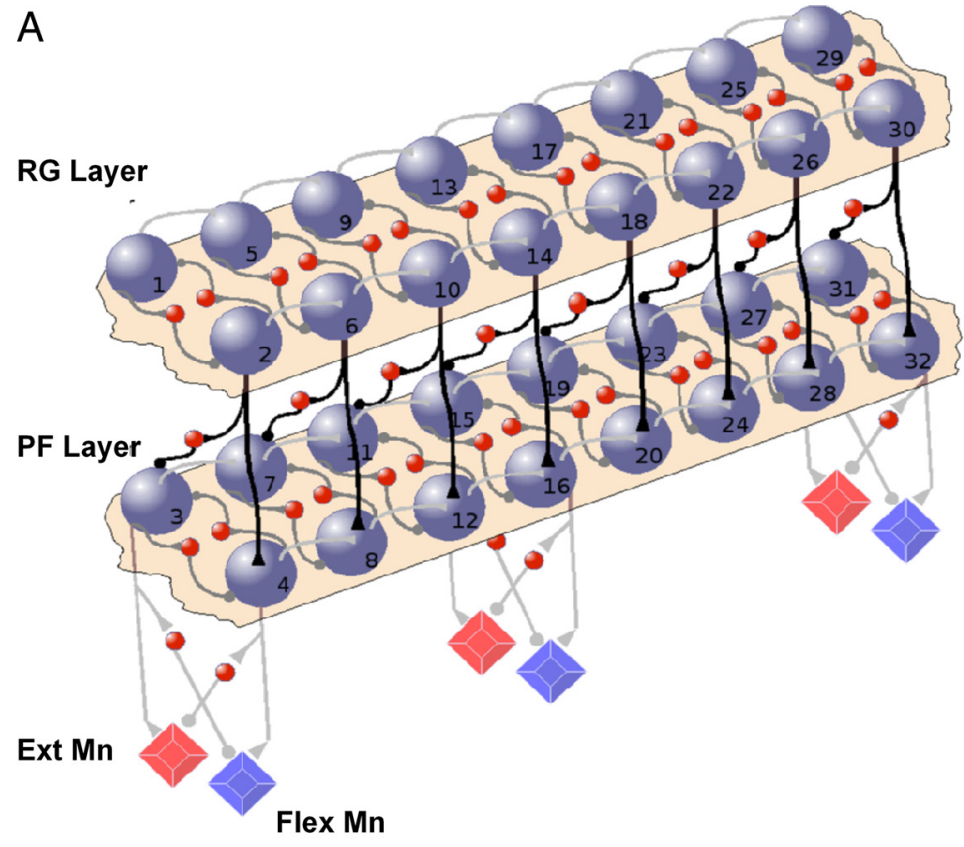

B

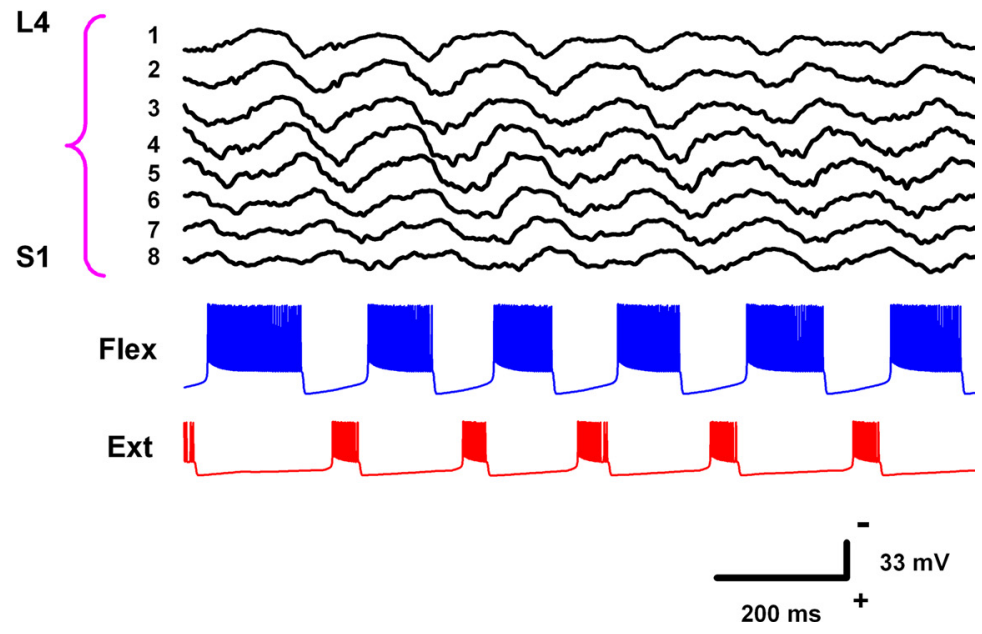

Figure 5. A, Schematic representation of the proposed intersegmental CPG network. Eight CPG nodes are assumed to be distributed along the spinal cord in an asymmetric two-layer organization. Each node contains populations of excitatory neurons (gray balls) and populations of inhibitory interneurons (small red balls) synaptically connected (gray and black lines). The light gray arrows within RG and PF layers denote synapses connecting neighboring nodes (motoneuron populations of nodes 2, 3, 5, 6 and 7 are omitted for a better visualization). $\boldsymbol{B}$, Propagation of the electrical activity along the spinal cord under normal operation (in the absence of induced deletions).

$$
\frac{d y}{d t}=\varepsilon\left(-\mu g_{\mathrm{Ca}} m_{\infty}(v)\left(v-V_{\mathrm{Ca}}\right)-y\right) .
$$

These equations describe the rate of change of the membrane potential $v(t)$, the slow recovering variable $w(t)$, and the calcium concentration $y(t)$. C represents the membrane capacitance per unit of area. This conductance-based model describes the dynamical behavior of the membrane potential $v(t)$, taking into account four ionic currents for the calcium, potassium, leakage, and calcium-dependent potassium channels with conductivities given by $g_{\mathrm{Ca}}, g_{\mathrm{K}}, g_{\mathrm{L}}$, and $g_{\mathrm{KCA}}$, respectively. With the inclusion of the calcium-dependent potassium channel the model qualitatively reproduces the bursting behavior observed experimentally. The dynamics of that channel is governed by the parameters $\mu$ and $\varepsilon$. The parameter $\mu$ is determined by the ratio between the surface area of the cell and the calcium volume. The parameter $\varepsilon$ is the product of the calcium degradation rate and the ratio of free to total calcium. Since calcium is usually neutralized, $\varepsilon$ is small and $y$ has a slow dynamics. Note that sodium channels are not essential to obtain a reasonable agreement with the experimental observations and consequently are not considered in the model. The nonlinear functions governing the dynamics of the ionic currents are given by the following:

$$
\begin{gathered}
m_{\infty}(v)=\frac{1}{2}\left(1+\tanh \left(\frac{v+V_{1}}{V_{2}}\right)\right) \\
w_{\infty}(v)=\frac{1}{2}\left(1+\tanh \left(\frac{v-W_{1}}{W_{2}}\right)\right) \\
\tau(v)=\cosh \left(\frac{v-W_{1}}{2 W_{2}}\right) \\
z(y)=\frac{y}{1+y},
\end{gathered}
$$

with $V_{1}, V_{2}, W_{1}$, and $W_{2}$ constants. $\phi$ is a parameter that represents the different time scales of the voltage membrane $v$ and the slow recovery variable $w$. The parameters $\varepsilon$ and $\mu$ are related to the duration and period of the burst, respectively.

The synaptic current of neuron $i$ is defined as described by Destexhe et al. (1994):

$$
I_{i}^{\text {syn }}=\sum_{j \in \text { neigh }(i)} g_{i j}^{\text {syn }} r_{j}\left(v_{i}-E_{\mathrm{s}}\right),
$$

where $g_{i j}^{\text {syn }}$ is the conductance of the synaptic channel, $E_{\mathrm{s}}$ is the synaptic rest potential (when larger than the neuron's rest potential the synapse is excitatory, and when smaller it is inhibitory), and $r_{j}$ represents the fraction of bound receptors described as follows:

$$
r_{j}=\left(1-e^{-\alpha t}\right) \quad \text { for } \quad t \leq t_{\mathrm{on}}
$$

$$
r_{j}=\left(1-e^{-\alpha t_{o n}}\right) \mathrm{e}^{-\beta\left(\mathrm{t}-\mathrm{ton}_{\mathrm{on}}\right)} \quad \text { for } \quad t>t_{\mathrm{on}},
$$

where $\alpha$ and $\beta$ are the rise and decay times respectively. Here, $t_{\text {on }}$ represents the time for which the synaptic connection is activated.

The parameters and synaptic connectivity values used in the simulations are listed in Tables 1 and 2, respectively. To evoke the incoming stimulation in the network, we increased the applied current of the neuron populations belonging to the RG layer of all the CPGs, and to neuron populations 3 and 4 , belonging to the PF layer, of the first CPG to $I=44.3 \mu \mathrm{A} / \mathrm{cm}^{2}$. The remaining neurons were subject to a constant bias current of $43.8 \mu \mathrm{A} / \mathrm{cm}^{2}$, which kept the neurons just below spiking threshold. 
Table 1. Parameters used in the numerical simulations

\begin{tabular}{lc}
\hline Parameter & Value \\
\hline$g_{\mathrm{Ca}}$ & $4.0 \mu \mathrm{S} / \mathrm{cm}^{2}$ \\
$g_{\mathrm{K}}$ & $8.0 \mu \mathrm{s} / \mathrm{cm}^{2}$ \\
$g_{\mathrm{L}}$ & $2.0 \mu \mathrm{S} / \mathrm{cm}^{2}$ \\
$g_{\mathrm{Kca}}$ & $0.25 \mu \mathrm{S} / \mathrm{cm}^{2}$ \\
$V_{\mathrm{Ca}}$ & $120.0 \mathrm{mV}$ \\
$V_{\mathrm{K}}$ & $-84.0 \mathrm{mV}$ \\
$V_{\mathrm{L}}$ & $-60.0 \pm 0.6 \mathrm{mV}$ \\
$V_{1}$ & $1.2 \mathrm{mV}$ \\
$V_{2}$ & $18 \mathrm{mV}$ \\
$W_{1}$ & $12 \mathrm{mV}$ \\
$W_{2}$ & $17.4 \mathrm{mV}$ \\
$\phi$ & $4.6 \mathrm{~s}^{-1}$ \\
$\varepsilon$ & $0.0175 \mathrm{~s}^{-1}$ \\
$\mu$ & $0.015-0.017$ \\
$l$ & $43.8 \mu \mathrm{AA} / \mathrm{cm}^{2}$ \\
$\alpha$ & $0.33 \mathrm{~ms}-1$ \\
$\beta$ & $0.2 \mathrm{~ms}$ \\
$E_{\mathrm{s}}$ & $0 \mathrm{mV},-80 \mathrm{mV}^{-1}$ (excitatory, inhibitory) \\
\hline
\end{tabular}

Table 2. Synaptic connectivity values

\begin{tabular}{lc}
\hline Connectivity & Value \\
\hline$g_{\text {exc,int }}$ & $0.3 \mu \mathrm{S} / \mathrm{cm}^{2}$ \\
$g_{\text {int,exc }}$ & $0.1-0.6 \mu \mathrm{S} / \mathrm{cm}^{2}$ \\
$g_{2,4}$ & $2.0 \mu \mathrm{S} / \mathrm{cm}^{2}$ \\
$g_{4, \text { flex }}$ & $2.0 \mu \mathrm{S} / \mathrm{cm}^{2}$ \\
$g_{3, \text { ext }}$ & $1.75 \mu \mathrm{S} / \mathrm{cm}^{2}$ \\
$g_{\text {int,flex }}$ & $1.5 \mu \mathrm{S} / \mathrm{cm}^{2}$ \\
$g_{\text {int,ext }}$ & $2.0 \mu \mathrm{S} / \mathrm{cm}^{2}$ \\
$g_{\text {exc,exc }}$ & $0.1 \mu \mathrm{S} / \mathrm{cm}^{2}$ \\
$g_{\text {CPG-CPG }}$ & $0.1 \mu \mathrm{S} / \mathrm{cm}^{2}$ \\
\hline
\end{tabular}

To account for experimental observations we allowed for different burst durations by varying the value of the parameter $\mu$ in the equation for $y$. As an example, neurons within populations 1, 3 , and extensor motoneurons of CPG1 in Figure $5 A$, and the equivalent ones in the other CPG units, have a value of $\mu=0.015$, which leads to an extensor phase of $\sim 70 \mathrm{~ms}$, whereas neurons within populations 2, 4, and flexor motoneurons, and the equivalent ones in the other CPG units, have a slightly larger value of $\mu=0.017$ for which the flexor phase lasts $\sim 140 \mathrm{~ms}$. The constructed network generates an alternating rhythm that propagates in the rostrocaudal direction, illustrated in Figure $5 B$, as observed experimentally (Cuellar et al., 2009; see also Bonnot et al., 2002; Yakovenko et al., 2002; Kaske et al., 2003; Ivanenko et al., 2006; Falgairolle and Cazalets, 2007).

\section{Resetting deletions associated with an abolition of the sinusoidal CDP}

Following the same procedure as Rybak et al. (2006b) we diminished temporally the currents in the RG and PF populations of all the nodes and observed a resetting deletion similar to the one found experimentally and shown in Figure 1. In our model, to evoke this deletion, we biased $50 \%$ of the neurons of the RG and PF population at $43.7 \mu \mathrm{A} / \mathrm{cm}^{2}$ during $400 \mathrm{~ms}$ whereas the other $50 \%$ remained at $44.3 \mu \mathrm{A} / \mathrm{cm}^{2}$. After this perturbation, the current of all the perturbed neurons was returned to its original values of $44.3 \mu \mathrm{A} / \mathrm{cm}^{2}$. Figure 6 shows the distortion of the sinusoidal CDP of the 8 nodes during a resetting deletion, indicating that it propagates longitudinally across the eight CPGs. Extensor motoneurons become silent due to the temporal lack of activity in the RG and PF layers whereas flexor motoneurons exhibit an increase of the bursting duration due to the absence of inhibition coming from PF layer [similar results were found in the experiments (Fig. $1 B)$ ].

\section{Non-resetting deletions associated with traveling sinusoidal CDPs}

To induce a non-resetting deletion in our model we suddenly diminished the bias current of neuron populations within the PF block of the CPGs. This sudden change was enough to generate a deletion that propagates along the segment. For example, when we diminished the applied current of $50 \%$ of the neurons of populations 3 and 4 to $43.7 \mu \mathrm{A} / \mathrm{cm}^{2}$ (the other $50 \%$ remains unaltered at $44.3 \mu \mathrm{A} / \mathrm{cm}^{2}$ ) during $100 \mathrm{~ms}$, we observed that neurons belonging to population 3 were silent, whereas the neurons of population 4 continued their bursting activity, due to the asymmetric connection between RG and PF layers. This lack of activity also affected the extensor motoneuron pool of the same CPG, whereas the activity of the flexor motoneuron pool remained unaffected as it is shown in Figure 7. This evoked deletion propagated across the longitudinal CPG modules, under the same current reduction of the subsequent PF nodes, as displayed in Figure 7. The two bottom traces of this figure clearly show that the deletion is reflected in absence of activity in the extensor motoneurons of the last CPG (node 8).

\section{Discussion}

In the present study we found that during non-resetting deletions in extensor activity the amplitude of the sinusoidal CDP spinal wave is not significantly affected. Furthermore, we found that resetting deletions were always associated with a concomitant absence of the CDP traveling spinal wave. Deletions of extensor activity and the sinusoidal-CDP traveling waves were reproduced with a numerical model of a network involved in the scratching rhythm based on the modified Morris-Lecar equations.

\section{Justification of use of the asymmetrical model of the CPG along the lumbosacral cord}

In the context of rhythm generation both the asymmetrical and non-asymmetrical models are hypothetical. However, there is strong experimental evidence supporting the asymmetrical model (Pearson and Duysens, 1976). Asymmetrical models in different species are based on the assumption that neurons with endogenous oscillatory capabilities activate exclusively flexor motoneurons, and that rhythmic activity in flexor motoneurons can be produced in the absence of extensor activity, but not the opposite. This suggests a lack of an extensor RG.

The location of the asymmetrical model proposed by Brownstone in upper lumbar segments is based on the location of candidate interneurons belonging to the RG (interneurons expressing the $\mathrm{Hb} 9$ transcription factor). There is no doubt that this small population of interneurons play a role in the rhythm generation of locomotion, or scratching, but we would be very surprised that they were the only type of interneurons with rhythm generation capabilities. There is evidence that lower lumbar and sacral isolated segments have rhythm generation capabilities too, therefore, different populations (Hb9 and non-Hb9 interneurons) must be playing the role of rhythm generators at all levels. The different classes of rhythm generation neurons might be coupled as a distributed system along the spinal cord. In fact there is evidence in neonatal mouse suggesting that $\mathrm{Hb} 9$ interneurons are unlikely to be the sole rhythm generating kernel of the CPG (Dietz et al., 2008). Consequently we believe that the asymmetric model may be applied to different spinal levels. However, there is 


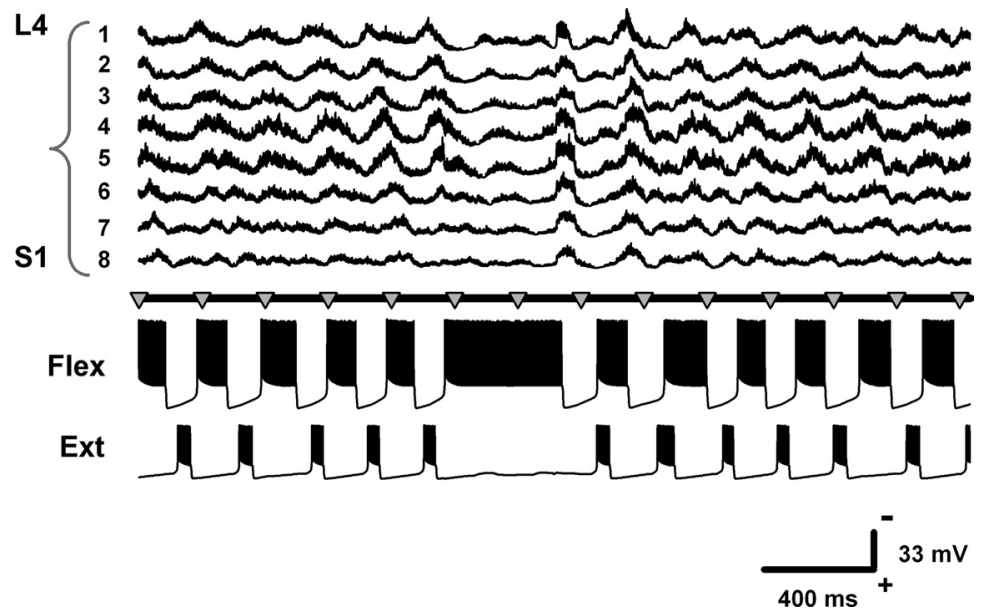

Figure 6. Example of a resetting deletion. A temporary lack of electrical activity ( $400 \mathrm{~ms}$ ) of the eight CPGs (from L4 to S1) and of the extensor motoneurons can be clearly seen. On the contrary, in flexor motoneurons the bursting period increases due to the lack of inhibition induced by the extensor group of the PF layer. Spaced arrowheads indicate the averaged period of the flexorextensor cycle before the deletion. Note the clear phase shift of the post-deletion rhythm.

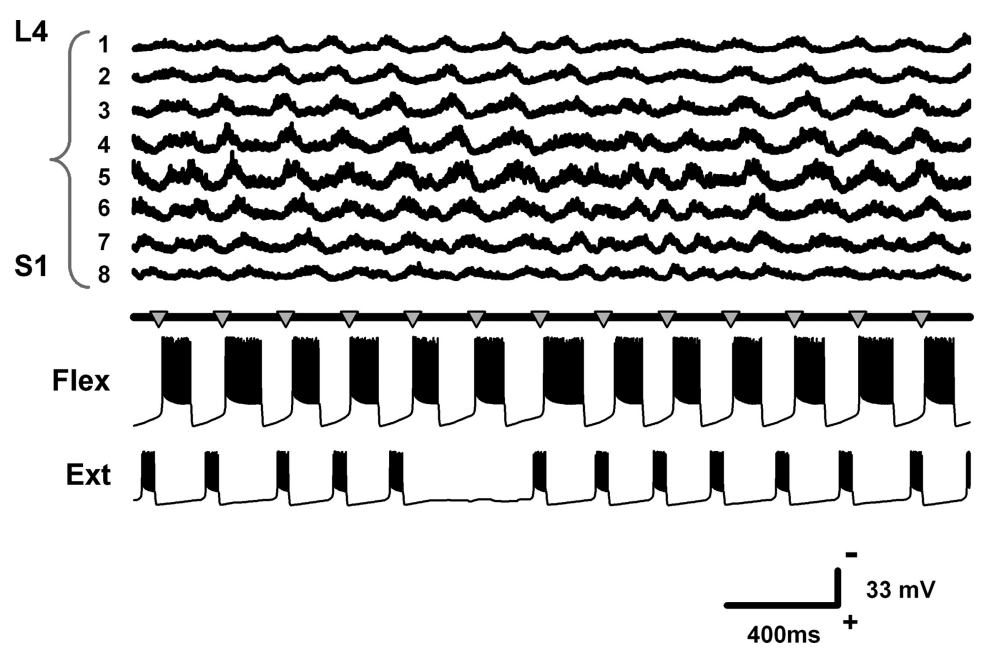

Figure 7. Example of a non-resetting deletion. The electrical activity of the eight $\mathrm{CPGs}$ (from L4 to S1) remains unaltered. On the contrary, extensor motoneurons become temporarily silent. In contrast to the resetting deletions during non-resetting deletions flexor motoneurons do not alter their bursting duration. Spaced arrowheads indicate the averaged period of the flexor-extensor cycle before the deletion. Note that there is not a phase shift of the post-deletion rhythm.

evidence (Endo and Kiehn, 2008) that last order extensor networks have also rhythm generation capabilities, although flexor networks dominate. This possibility sounds like a plausible idea, but to what extent this organization occurs at the rhythm generation level is unknown.

Based on quantitatively conductance analysis the experiments by Endo and Kiehn (2008) show elegantly that the flexor-related and extensor-related last order interneurons are asymmetrically, but still, reciprocally organized. Our PF network is reciprocally organized and reproduces the pattern of rhythmic activity during scratching. These facts justify the reciprocal organization of the PF layer in our model (Fig. 5).

Non-resetting and resetting deletions: Possible origin In the present study we observed some episodes with nonresetting deletions followed by resetting deletions, but for clarity and simplification of the model, we only analyzed episodes with only one class of deletion (resetting or non-resetting). Nonresetting deletions represent absences in the firing of the mo- toneurons without phase shifts in the cycle. These deletions have been observed during locomotion and scratching in the cat (Duysens, 1977; Grillner and Zangger, 1979; Jordan, 1991; LafreniereRoula and McCrea, 2005) and during scratching in the turtle (Stein, 2005). A plausible explanation for these deletions is that the CPG controlling the motoneuron activity contains a neuronal network capable of maintaining the rhythm even during a deletion in which a lack of the motoneuron activity is observed.

Figures 2 and $3 B$ show that traveling spinal waves persist during non-resetting deletions of expected burst in extensor activity, thus suggesting the existence of a traveling master-clock constituted by groups of neurons longitudinally distributed and sequentially activated from the L4 to S1 spinal segments. This finding indicates that during scratching there is a sequential order of activation of spinal neurons, presumably central-rhythmgenerator neurons from rostral to caudal segments, acting on the pattern formation network. This hypothesis is compatible with the two-layer architecture of the CPG (McCrea and Rybak, 2008) and with the longitudinal architecture of the unit burst generators (Grillner, 1981).

We found sinusoidal-CDPs during non-resetting deletions in extensor activity (Fig. 3B). Furthermore, our model shows that the non-resetting deletions can be produced by a failure at the level of the extensor PF layer (LafreniereRoula and McCrea, 2005). These results suggest that the extensor PF network and the extensor motoneurons may not contribute to the generation of the sinusoidalCDP during non-resetting deletions. Therefore, it is tempting to speculate that the populations of interneurons active during a non-resetting deletion belong to the flexor RG layer (an element of the master clock), or to a synchronized population of spinal interneurons following the rhythmic command from the master clock ("followers different to the PF neurons"). During non-resetting deletions we can exclude the only participation of flexor PF neurons in the generation of the sinusoidal-CDP because the rhythm does not change during these deletions (an RG must be present).

We only observed deletions of extensor activity (but not of flexor activity) during fictive scratching. This could be explained by the fact that the RG is of flexor nature according to the Brownstone's asymmetric model of the CPG. Therefore, resetting deletions would occur only when there is a change in the timing of the $\mathrm{RG}$ rhythm (that is, flexion), which is the timing imposed to extensor and flexor PF networks. Thus because of the flexor nature of the RG, it is not possible for resetting extensor deletions to occur, as is the case for scratching.

As shown in Figures 6 and 7, many of the features exhibited by the CPGs can be reproduced by a longitudinal and asymmetrical 
model of the CPG. Our model is a combination of those proposed by Rybak and McCrea (2006b) and Brownstone and Wilson (2008) in the scheme of the two-layer model of CPG, and Grillner (2006) in the context of the rostrocaudal propagation of the spinal electrical activity during scratching. For example, the rostrocaudal propagation of the CDP obtained with our model (Fig. $5 B$ ) is consistent with the rostrocaudal propagation of the traveling spinal wave during scratching (Cuellar et al., 2009, their Fig. 1). Based on our intersegmental (longitudinal) two-layer model of the CPG, we suggest that resetting deletions correspond to a failure at the longitudinal level of both the rhythm-generation and pattern-formation layers, whereas non-resetting deletions represent a failure at the longitudinal level of the pattern formation network. In this context, our study extends the model of the two-layer organization of the CPG described in one single spinal segment, thus providing new clues for their longitudinal spatiotemporal organization. The model includes an intrinsic diversity in the parameters of the neurons forming the CPG, to represent the natural heterogeneity present in the real system. Such diversity can be expected to lead to more robust operation of the system under variation of external conditions, due to the averaging of the neuronal responses. Diversity has also been proposed as a source of regular behavior in nonlinear systems (Tessone et al., 2006).

We observed that after the ending of the resetting deletions, sinusoidal waves resume synchronously both experimentally (Fig. $1 B$, vertical rectangle) and theoretically (Fig. 6), but it can take one or more cycles for the phase gradient to appear. A plausible interpretation for the experimental data can be given in terms of the model. The synchronized timing of the propagating waves after the resetting deletions reflects the end of the failure at the level of the whole RG and PF networks. This failure could be associated with a global change in the excitability of the whole CPG. In our simulations, this effect is modeled by a sudden change from the perturbed (subthreshold) value to the initial (suprathreshold) value of the applied current of the neurons belonging to both RG and PF layers. Consequently, after the perturbation all neurons start spiking initially at the same time, but subsequently the alternating cycle is recovered because of the longitudinal architecture of the CPG. Our results are explained on the base of an asymmetric model assuming only flexor RG. If flexor activity becomes tonic there will always be a tonic inhibition of the extensor PF half center (Fig. 5A), accompanied of extensor resetting deletions and absence of CDP sinusoidal waves (Fig. 1, deletion indicated by d). On the other hand, as can be seen in Figures 2, 3B, and 7, extensor non-resetting deletions are accompanied by unaltered CDP sinusoidal waves and an "absence" (due to rhythmic alternation) of activity in the flexor nerves. Based on the asymmetrical model, in this last case rhythmic activity is occurring in the RG and the non-resetting extensor deletions are taking place at the PF level.

Our results support the hypothesis that within the spinal cord there is a group of interneurons longitudinally organized as shown in Figure $5 A$, that are capable of maintaining the CPG rhythm even during deletions in the extensor motoneuron activity. We suggest that the traveling electrical waves in the lumbosacral spinal cord during scratching (recently reported by Cuellar et al., 2009) are produced by the sequential activation of those circuits.

\section{References}

Barajon I, Gossard JP, Hultborn H (1992) Induction of fos expression by activity in the spinal rhythm generator for scratching. Brain Res 588:168-172.

Bayev KV, Kostyuk PG (1981) Primary afferent depolarization evoked by the activity of spinal scratching generator. Neuroscience 6:205-215.
Berkinblit MB, Deliagina TG, Feldman AG, Gelfand IM, Orlovsky GN (1978) Generation of scratching. I. Activity of spinal interneurons during scratching. J Neurophysiol 41:1040-1057.

Bonnot A, Whelan PJ, Mentis GZ, O’Donovan MJ (2002) Locomotor-like activity generated by the neonatal mouse spinal cord. Brain Res Rev 40:141-151.

Brown TG (1911) The intrinsic factor in the act of progression of the mammal. Proc R Soc Lond B Biol Sci 84:308-319.

Brownstone RM, Wilson JM (2008) Strategies for delineating spinal locomotor rhythm-generating networks and the possible role of $\mathrm{Hb} 9$ interneutones in rhythmogenesis. Brain Res Rev 57:64-76.

Burke RE, Degtyarenko AM, Simon ES (2001) Patterns of locomotor drive to motoneurons and last-order interneurons clues to the structure of the CPG. J Neurophysiol 86:447-462.

Cuellar CA, Tapia JA, Juárez V, Quevedo J, Linares P, Martínez L, Manjarrez E (2009) Propagation of sinusoidal electrical waves along the spinal cord during a fictive motor task. J Neurosci 29:798-810.

Deliagina TG, Orlovsky GN, Perret C (1981) Efferent activity during fictitious scratch reflex in the cat. J Neurophysiol 45:595-604.

Destexhe A, Mainen Z, Senjowski T (1994) An efficient method for computing synaptic conductances based on a kinetic model of receptor binding. Neural Comput 6:14-18.

Dietz S, Kwan AC, Webb WW, Harris-Warrick RM (2008) Hb9 interneuron activity during fictive locomotion in mouse spinal cord. Soc Neurosci Abstr 34:374.17.

Duysens J (1977) Reflex control locomotion as revealed by stimulation of cutaneous afferents in spontaneously walking premammillary cats. J Neurophysiol 40:737-751.

Duysens J, Pearson KG (1980) Inhibition of flexor burst generator by loading ankle extensor muscles in walking cats. Brain Res 187:321-332.

Endo T, Kiehn O (2008) Asymmetric operation of the locomotor central pattern generator in the neonatal mouse spinal cord. J Neurophysiol 100:3043-3054

Falgairolle M, Cazalets JR (2007) Metachronal coupling between spinal neuronal networks during locomotor activity in newborn rat. J Physiol $580: 87-102$.

Grillner S (1981) Control of locomotion in bipeds, tetrapods and fish. In: Handbook of physiology. The nervous system II. Motor control (Brooks VB, ed), pp 1179-1236. Baltimore: Waverly.

Grillner S (1985) Neurobiological bases of rhythmic motor acts in vertebrates. Science 228:143-149.

Grillner S (2006) Biological pattern generation: the cellular and computational logic of networks in motion. Neuron 52:751-766.

Grillner S, Wallén P (1985) Central pattern generators for locomotion, with special reference to vertebrates. Annu Rev Neuroci 8:233-261.

Grillner S, Zangger P (1979) On the central generation of locomotion in the low spinal cat. Exp Brain Res 34:241-261.

Ivanenko YP, Poppele RE, Lacquaniti F (2006) Spinal cord maps of spatiotemporal alpha-motoneuron activation in humans walking at different speeds. J Neurophysiol 95:602-618.

Jordan LM (1991) Brainstem and spinal cord mechanisms for the initiation of locomotion. In: Neurobiological basis of human locomotion (Shimamura M, Grillner S, Edgerton VR, eds), pp 3-20. Tokyo: Japan Scientific Societies/Springer.

Kaske A, Winberg G, Cöster J (2003) Emergence of coherent traveling waves controlling quadruped gaits in a two-dimensional spinal cord model. Biol Cybern 88:20-32.

Kiehn O (2006) Locomotor circuits in the mammalian spinal cord. Annu Rev Neurosci 29:279-306.

Kiehn O, Kjaerulff O (1998) Distribution of central pattern generators for rhythmic motor outputs in the spinal cord of limbed vertebrates. Ann N Y Acad Sci 860:110-129.

Kriellaars DJ, Brownstone RM, Noga BR, Jordan LM (1994) Mechanical entrainment of fictive locomotion in the decerebrate cat. J Neurophysiol 71:2074-2086

Lafreniere-Roula M, McCrea DA (2005) Deletions of rhythmic motoneuron activity during fictive locomotion and scratch provide clues to the organization of the mammalian central pattern generator. J Neurophysiol 94:1120-1132.

McCrea DA, Rybak IA (2008) Organization of mammalian locomotor rhythm and pattern generation. Brain Res Rev 57:134-146. 
Orlovsky GN, Deliagina TG, Grillner S (1999) Neuronal control of locomotion. From mollusk to man. New York: Oxford UP.

Pearson KG (1972) Central programming and reflex control of walking in the cockroach. J Exp Biol 56:173-193.

Pearson KG, Duysens JD (1976) Function of segmental reflexes in the control of stepping in cockroaches and cats. In: Neural control of locomotion (Herman RM, Grillner S, Stein PSG, Stuart DG, eds), pp 519-538. New York: Plenum.

Pearson KG, Iles JF (1970) Discharge patterns of coxal levator and depressor motoneurons of the cockroach, Periplaneta americana. J Exp Biol 52:139-165.

Perret C, Cabelguen JM (1976) Central and reflex participation in the timing of locomotor activations of a bifunctional muscle, the semitendinosus, in the cat. Brain Res 106:390-395.

Perret C, Cabelguen JM (1980) Main characteristics of the hindlimb locomotor cycle in the decorticate cat with special reference to bifunctional muscles. Brain Res 187:333-352.

Perret C, Cabelguen JM, Orsal D (1988) Analysis of the pattern of activity in "knee flexor" motoneurons during locomotion in the cat. In: Stance and motion: facts and concepts (Gurfinkle VS, Ioffe ME, Massion J, Roll JP, eds) pp 133-141. New York: Plenum.
Rinzel J, Ermentrout GB (1998) Analysis of neural excitability and oscillations. In: Methods in neuronal modelling: from ions to networks (Koch C, Segev I, eds), pp 251-291. Cambridge, MA: MIT.

Rybak IA, Shevtsova NA, Lafreniere-Roula M, McCrea DA (2006a) Modelling spinal circuitry involved in locomotor pattern generation: insights from deletions during fictive locomotion. J Physiol 577:617-639.

Rybak IA, Stecina K, Shevtsova NA, McCrea DA (2006b) Modelling spinal circuitry involved in locomotor pattern generation: insights from the effects of afferent stimulation. J Physiol 577:641-658

Sherrington CS (1906) Observation of the scratch-reflex in the spinal dog. J Physiol 34:1-50.

Sokal R, Rohlf J (1969) Biometry. The principles and practice of sctatistics in biological research. San Francisco: Freeman.

Stein PS (2005) Neuronal control of turtle hindlimb motor rhythms. J Comp Physiol A Neuroethol Sens Neural Behav Physiol 191: 213-229.

Tessone CJ, Mirasso CR, Toral R, Gunton JD (2006) Diversity-induced resonance. Phys Rev Lett 97:194101.

Yakovenko S, Mushahwar V, VanderHorst V, Holstege G, Prochazka A (2002) Spatiotemporal activation of lumbosacral motoneurons in the locomotor step cycle. J Neurophysiol 87:1542-1553. 